\title{
HABITAT JUVENIL EN LA METROPOLI: JUVENTUD Y TERRITORIO
}

\author{
CAROLINA TKACHUK ${ }^{*}$
}

\section{PRESENTACión}

¿CUÁNTA IMPORTANCIA TIENEN LOS jóvenes para la ciudad y cuánta tiene la ciudad para ellos?, ¿qué es la ciudad para los jóvenes?, ¿qué son los jóvenes para la ciudad? En un intento de vincular a los actores sociales con la gestión urbana, la idea que aquí me propongo es tomar un subgrupo específico de actores, los jóvenes, e indagar el grado de consideración que ellos tienen a través de las políticas de juventud que surgen —o no- desde los distintos ámbitos de gobierno (municipal, metropolitano, provincial, nacional). Para ello, nos concentraremos en la creación de espacios y producción del hábitat para la juventud en la ciudad, así como en la instalación de espacios de consumo urbano y cultural en franjas juveniles, como una manera de ver y analizar el rol asignado a los jóvenes en la agenda de políticas públicas, o sea, desde la gestión, al momento de pensar la ciudad, delinear un modelo de ciudad, construir una imagen futura de ciudad. En definitiva, lo que estamos intentando develar es el grado de interacción (social y territorial) existente entre los jóvenes y la ciudad.

* Licenciada en Comercio Internacional de la Universidad Nacional de Quilmes. Posgrado en Economía Urbana en la Universidad Torcuato Di Tella (en curso). Investigadora del Proyecto: «Construir-Habitar-Pensar: nuevas modalidades de producción y gestión de la RMBA en el nuevo milenio».

E-Mail: ctkachuk@unq.edu.ar. 
Por otro lado, como eje central de las condiciones espaciales y de hábitat en las que se inserta cada uno de los actores urbanos que conforman la ciudad, la idea también aquí es considerar la relevancia que adquiere la vivienda para dichos sujetos, concentrándonos - en nuestro caso- en las implicancias y desafíos existentes para los jóvenes, indagando sobre las nuevas y complejas condiciones de acceso a la vivienda de los sectores juveniles, sobre circunstancias que hacen posible un debate en torno a la cuestión habitacional instalando a la vivienda como instrumento emancipador por un lado, y de integración social a la ciudad, por otro.

\section{LOS JÓVENES COMO ACTORES SOCIALES}

Desde el momento en que podemos pensar la ciudad como sociedad que adopta un determinado sistema de relaciones entre actores, donde las dimensiones sociales y territoriales están determinando comportamientos sociales urbanos, se observa que la relación entre actores y territorio urbano tiende a ser estrecha. Esto nos permite identificar una doble concepción de ciudad: aquella que declara la ciudad como variable dependiente, en donde los procesos sociales explican gran parte de la configuración urbana del territorio; y otra, que concibe a la ciudad como variable independiente, cuando esos procesos sociales son entendidos a través de los fenómenos urbanos.

Los jóvenes son concebidos actores sociales por ser sujetos reales de acción cuyo comportamiento se relaciona con las estructuras de la sociedad (base social) a la vez que están sometidos a ciertas estrategias que van en función de sus propios intereses culturales, políticos, territoriales, etc.; su comportamiento se determina en función de una lógica local al tiempo que ese comportamiento está también determinando ciertos procesos locales; se trata de actores que se reproducen como tales dentro del ámbito local.

Resulta interesante considerar, sobre todo concentrándonos en el análisis de los jóvenes y su territorio, cierta definición de actores urbanos en función del hábitat específico en la ciudad que se genera desde los propios actores y que a la vez se configura sobre un conjunto de características inherentes a ellos, ya sea la relación entre la reproducción socioeconómica y el espacio (entendiendo que cada actor se reproduce cuando el desarrollo de sus actividades le permite mantenerse, desarrollarse y crecer como personaje urbano), las distintas formas de asentamientos en relación con el suelo y la vivienda, la 
relación existente con el territorio urbano construido, etc. Esto da lugar a la generación de identidades que guardan una estrecha vinculación con el espacio que se habita, lo que nos permite entender al territorio como un espacio trascendental que representa la identidad de sus actores, y que supone una condición de reproducción de grupos específicos; la autoidentificación de los individuos está relacionada con el lugar que ocupan.

Actores y territorio aparecen interrelacionados por medio de la identidad, categoría que — lejos de ser estática — tiende a ser dinámicamente evolutiva.

\section{BUENOS AIRES JOVEN: ESPACIOS URBANOS DE VANGUARDIA}

Es interesante observar el surgimiento de algunos barrios en la Ciudad de Buenos Aires que se han «recreado» con una clara impronta juvenil, con usos predominantemente culturales, de entretenimiento y de reencuentro social; este proceso urbano-social conlleva muchas veces a una cierta redefinición en cuanto a la denominación de los barrios, aludiendo muchas veces a algún estilo social particular. El típico caso se ubica, por ejemplo, en la zona de Palermo en sus variantes «Palermo Hollywood» y «Palermo Soho», donde puede verse un conglomerado de lugares de diseño, exposiciones de arte, locales de ocio y entretenimiento, configurando un circuito juvenil donde la vanguardia y la imagen convalidan un área con una estética, prácticas y códigos propios. Basta un adjetivo para entender el estilo del lugar, estilo que advierte una nueva cultura «callejera» de estar en la ciudad frente a las tendencias de «hermética urbana» propuestas por los shoppings a lo largo de los años noventa; cultura que pasea por los barrios, por las calles, construyendo nuevos significados en nuevos espacios y creando a su vez nuevas identidades en lugares con cierta impronta cosmopolita a tono con el mundo, pero proponiéndose una mirada local. En este sentido, es interesante revelar que estas transformaciones sociales y territoriales que surgen dentro de un área determinada de Buenos Aires, se plasman también sobre el circuito económico que le permite reproducirse como territorio urbano; los nuevos usos del suelo que se van generando reestructuran al mismo tiempo las relaciones económicas urbanas del lugar. 


\section{IMPACTOS DE LA GLOBALIZACIÓN EN EL HÁBITAT JUVENIL (Hacia un proceso de creación de espacios para jóvenes urbanos)}

Las políticas neoliberales adoptadas durante la década de los noventa - con una clara impronta globalizadora- suponen un proceso de transformación socio-territorial sobre tres ejes interrelacionados: en primer lugar, sobre las formas de gestión urbana tendiendo a continuar y respetar lineamientos «globales» en el desarrollo de las políticas urbanas; en la fisonomía de la ciudad; en las prácticas, costumbres y acciones de sus habitantes, como así también sobre los modos de habitar que se adquieren sobre distintos ángulos territoriales de la metrópoli.

La llegada de inversiones extranjeras a lo largo de la última década se materializa territorialmente a través de la generación de espacios urbanos dualizados, profundizando las tendencias de segregación social y territorial, advirtiendo una doble velocidad en el desarrollo urbano, proceso que podemos observar - por ejemplo - mediante la yuxtaposición de «zonas atrasadas» (marginales) y zonas modernas dentro del ámbito metropolitano. Un caso ilustre en este sentido puede verse en la localización de muchos barrios privados al límite de villas miseria. Lo que se observa es que la selectividad territorial del capital (inversiones) retroalimenta estos movimientos contradictorios de estructuración territorial y, por ende, lo agudiza, con claros impactos sobre el hábitat que se despliega a lo largo de la ciudad.

Casualmente, uno de los espacios urbanos gravitacionales de gran parte de las inversiones externas lo integran los grandes centros comerciales y recreativos estrechamente vinculados al hábitat juvenil, capitales que van exodirigidos de manera directa hacia el foco y epicentro de socialización, de reencuentro donde se despliegan diversas prácticas de identificación y realización propia de la población juvenil.

La relación entre juventud y territorio se vuelve una relación entre juventud y sociedad desde el momento en que las transformaciones producidas en el conglomerado metropolitano durante los noventa advierten el surgimiento de espacios de apropiación colectiva en el medio urbano de socialización juvenil donde sus integrantes encuentran obstáculos en su tránsito hacia roles adultos. Este fenómeno es entendido por el restringido acceso de los jóvenes a los servicios educativos, laborales, de vivienda, entre otros, deteriorando así el sentido de pertenencia social que es percibido desde la juventud. De esta manera, se intenta reflejar a los jóvenes como actores sociales margina- 
dos, con un conjunto de bienes y servicios a los cuales su acceso se torna complejo.

\section{DUALIDAD EN LOS ESPACIOS JUVENILES}

La configuración dual de espacios urbanos, sobre la que hicimos referencia anteriormente, como una de las tendencias territoriales que se ha venido gestando durante esta última década, donde la modernidad nace paralelamente con zonas marginales, también puede verse plasmada al interior de lo que estamos considerando como hábitat juvenil, desde el momento en que estos espacios - fundamentalmente las grandes superficies comerciales y recreativas- emergen como «pequeños suburbios» de la ciudad, donde hacia adentro se internalizan recursos permitiendo a los jóvenes clasificarse y clasificar a los demás, en función de un consumo urbano determinado. Lo que se intenta reflejar es que aquella «dualidad territorial» que se despliega sobre la metrópoli en general puede verse circunscripta simultáneamente como un caso particular de este proceso en los ámbitos de concurrencia juvenil.

A partir de aquí, la expresión juvenil sobre el territorio ya no es la misma para todos; los modos y las vías de acceso a estos espacios por parte de cierto sector juvenil se vuelve restrictivo dificultando procesos de integración social. Por otro lado, los jóvenes de los sectores medios y altos tienden a adquirir un consumo mucho más directo del espacio, no sólo desde la dimensión material (tangible) mediante el consumo de bienes y servicios que son ofrecidos, sino también desde una dimensión simbólica (intangible) a través de sus prácticas sociales, signos y códigos visuales o corporales que se despliegan sobre aquellos espacios de ocio, reencuentro y socialización.

Y entonces ¿cuál es el nuevo rol del territorio? Nos queda claro que se presenta como la reproducción de la interrelación y dialéctica de fuerzas económicas, sociales, políticas y culturales. Pero al mismo tiempo nos obliga a reflexionar sobre las nuevas formas de producción del hábitat juvenil tendiendo a resignificar los elementos que integran el medio construido, tanto por parte de aquellos que lo planifican y gestionan, pasando por quienes ofrecen sus bienes y servicios y, fundamentalmente, por parte de quienes están demandando el espacio, que son específicamente los jóvenes. Finalmente, la creación y producción de complejos juveniles integrando la regulación del uso del espacio y tendencias socioeconómicas locales amerita verificar necesidades y aspiraciones de los actores sociales que conforman el territorio urbano. 


\section{EL ESPACIO DES-HABITADO POR LOS JÓVENES: LA VIVIENDA PROPIA}

En primer lugar, resulta oportuno y necesitamos plantear una delimitación conceptual respecto de la noción de juventud que se toma a los fines del presente análisis. Si bien no existe un criterio único, se refiere al período de vida en que las personas transitan de la niñez a la condición de adulto; la extensión de este período varía según las sociedades, las culturas, las clases sociales y el género. El rango de edad utilizado como criterio para identificar a la población joven establece la edad de entrada en función de cuestiones biológicas y psicológicas, en la que ya están desarrolladas las funciones sexuales y reproductivas, que diferencian con claridad al adolescente del niño y tienen profundas repercusiones en su dinámica física y biológica. En tanto, la cota superior se identifica con el momento en que los individuos alcanzan cierta madurez social, es decir, al cierre del ciclo educativo formal, enfrentando el ingreso al mercado de trabajo y formación de un hogar propio. La juventud es así definida como el conjunto de individuos de la población que se ubica entre los 15 y los 29 años (Fernández, 2003).

La política de vivienda debería entender a la vivienda, principalmente, como un derecho al goce del hábitat que tienen todos los individuos, integrando este derecho incluso la autoidentificación como ciudadanos en sus prácticas sociales, mediante una participación que se ve plasmada a través de la inserción institucional genuina a la comunidad a partir del acceso a la vivienda, lo que a su vez contribuye a la generación de capacidades para la formación y consolidación de identidades individuales.

Más allá de las diversas concepciones que giran en torno a la vivienda, en principio debería ser entendida como instrumento de desarrollo personal y de integración social, involucrando no sólo elementos materiales (recursos, tecnología, etc.), sino también considerando necesidades, características y cultura de quien la habitará. Así, la vivienda concebida como lugar para morar, para establecer raíces, para identificarse como individuo y como ciudadano apunta al mejoramiento de la calidad de vida, desde una perspectiva interdisciplinaria y sobre un sistema dialéctico de necesidades y satisfactores (Fernández, 2003). Tal como declara Yujnovsky, «derecho a la vivienda como un objetivo social y político, pero también ético de justicia social, que persiguen las sociedades realmente democráticas» (Yujnovsky, 1984:18); donde tras- 
ciende la noción de vivienda al mero objeto-mercancía con una marcada tendencia a entender la vivienda como hábitat de relaciones sociales.

Tradicionalmente, la problemática habitacional ha sido abordada bajo la definición de acciones centralizadas, con sistemas decisionales cerrados y con un restringido componente de participación de los beneficiarios. En tanto, bajo prácticas más vinculadas con los valores y responsabilidades sociales pueden definirse políticas con formas alternativas de proyección (por ejemplo, con participación de la comunidad), dando cuenta de una mayor necesidad de un sistema abierto de decisiones, descentralizado, con espacios hegemónicos de participación al momento de concretar soluciones habitacionales para grupos específicos, en nuestro caso los jóvenes.

Esta consideración se ubica en línea con lo que postula Yujnovsky, «la vivienda es una configuración de servicios - habitacionalesque deben dar satisfacción a necesidades humanas primordiales: albergue, refugio, protección ambiental, espacio, vida de relación, seguridad, privacidad, identidad, accesibilidad física, entre otras. Estas necesidades varían con cada sociedad y grupo social y se definen en el devenir histórico. La producción de servicios habitacionales, así como la política de vivienda, tiene lugar en una sociedad determinada, con una cierta organización social y relaciones de poder. Por lo tanto, las condiciones de vivienda y la política habitacional sólo pueden analizarse teniendo en cuenta las diversas estructuras y relaciones de la sociedad y el Estado» (Yujnovsky, 1984:17); propiciando una estructura abierta y flexible entre la comunidad y el gobierno en lo que respecta a la satisfacción de las necesidades de vivienda.

\section{LA VIVIENDA: REVELADORA DE LA CALIDAD DE VIDA}

Teniendo siempre presente la relevancia social que adquiere para un individuo el acceso a la vivienda propia, podemos entender que la satisfacción - concebida como ingrediente sustancial de la calidad de vida - depende de las evaluaciones que el individuo lleva a cabo sobre los atributos de cada uno de los ámbitos, dominios o componentes de su existencia. En este sentido, lo que estaría determinando la calidad de vida es la interacción entre lo subjetivo y lo objetivo, en nuestro caso entre el sujeto y la vivienda. A partir de aquí cabría evaluar hasta qué punto las necesidades de vivienda están directamente vinculadas con la disponibilidad de recursos económicos; es decir, entendemos que el acceso a la vivienda se encuentra fuertemente determi- 
nado por los recursos que el sujeto dispone, pero también entendemos que trasciende el plano económico en el momento en que se revelan las verdaderas necesidades y expectativas que promueven una idea de vivienda propia. En ese momento, el determinante fundamental de la calidad de vida individual es el «ajuste» entre las características de la situación (involucrando exigencias y oportunidades) y las expectativas, capacidades y necesidades del sujeto tal y como éste las percibe.

\section{Cuadro 1}

Grado de importancia de factores constitutivos de la calidad de vida

\begin{tabular}{|c|l|}
\hline $\begin{array}{c}\text { ORDEN DE IMPORTANCIA } \\
\text { (EN PROMEDIO) }\end{array}$ & \multicolumn{1}{c|}{ FACTORES } \\
\hline $1^{\circ}$ & Posibilidad de educarse. \\
\hline $2^{\circ}$ & Inexistencia de contaminación urbana. \\
\hline $3^{\circ}$ & Accesibilidad a la salud. \\
\hline $4^{\circ}$ & $\begin{array}{l}\text { Respeto por los derechos cívicos. } \\
\text { Posibilidad de disponer de una vivienda adecuada. }\end{array}$ \\
\hline $5^{\circ}$ & Buen funcionamiento de servicios públicos. \\
\hline $6^{\circ}$ & Posibilidad de participación sociopolítica. \\
\hline $7^{\circ}$ & Posibilidad de trabajar en lo que se quiera. \\
\hline $8^{\circ}$ & Posibilidades de diversión. \\
\hline
\end{tabular}

Fuente: elaboración propia en base a datos del INDEC.

Con el objetivo de identificar y analizar la percepción de los jóvenes de la Ciudad de Buenos Aires acerca de determinados aspectos vinculados con la calidad de vida, se llevó a cabo un estudio analítico a título comparativo en base a un conjunto de datos estadísticos obtenidos a través de una serie de encuestas realizadas por INDEC sobre una franja juvenil de 15 a 20 años durante el año 1999. La incorporación de indicadores subjetivos para evaluar la calidad de vida, es abordada desde una perspectiva actitudinal incluyendo aspectos cognitivos, afectivos y conductales. Los resultados obtenidos del estudio están reflejados en el cuadro anterior.

Los datos precedentes revelan la importancia que los jóvenes otorgan a un conjunto de factores determinantes de la calidad de vida, priorizando la educación, la preservación del medio ambiente y la salud, antes que las posibilidades de acceder a una vivienda propia, dando cuenta del escaso nivel de preocupación que demuestran por el acceso a un bien y a un activo que en ningún momento deja de ser una de sus principales prioridades. Es interesante también destacar una 
cuestión que se vincula con el momento en que muchas veces el acceso a una vivienda comienza a ser una preocupación en los jóvenes, un momento que equivale al instante en que los jóvenes deciden iniciar una vida en pareja (legal o de hecho), instalando la igualdad vivienda = pareja, sin concebir a la vivienda como una etapa de desarrollo personal ex-ante.

La escasa relevancia en torno a la vivienda percibida por los jóvenes se hace más evidente todavía si continuamos en la lectura de los datos y observamos el bajo interés que otorgan a las posibilidades de participar políticamente frente a un conjunto de problemáticas en las que se encuentran incluidos y que, por lo tanto, les compete.

No obstante, hay que considerar que esta crisis de participación se deriva de la ineficiencia de las instituciones como mecanismos de mediación entre las demandas de diversos sectores y los centros de decisión, produciendo comportamientos de retraimiento y entorpeciendo la formación de estructuras de representación frente a las cuales los jóvenes puedan sentirse identificados.

\section{DESAFÍOS}

Una nueva cultura de lucha por el derecho a la ciudad se ubica en la instancia del denominado cooperativismo autogestionario que supone ser una práctica muy arraigada por sectores populares en los últimos años al momento de encarar la política habitacional en algunos barrios de la Ciudad de Buenos Aires, demostrando una gran capacidad de organización y definición de objetivos colectivos comunes, como propuesta para disputar el territorio y la producción del hábitat urbano desde un protagonismo popular y como herramienta de construcción de poder endógeno conjuntamente con participación estatal.

Como fuimos develando, esta práctica, que nace desde la misma comunidad y sectores populares que declaran explícitamente una necesidad habitacional, no se refleja de la misma manera en los sectores juveniles quienes aún con el peso del déficit habitacional a cuestas, no parecieran manifestar cierta preocupación por la vivienda como meta de desarrollo personal, ni siquiera como derecho a habitar la ciudad.

Lo que se observa es más bien una ausencia de actitudes y expectativas en cuanto a la participación y el trabajo colectivo, sin lograr aunar intereses frente a una cuestión que compete a la mayoría de ellos; esa falta de sentido del derecho a la ciudad da cuenta de la falta de valorización del espacio urbano como espacio social y revela in- 
tersticios en cuanto a los modos de pensar la ciudad. En este sentido, no tiende a existir un grado creciente de representaciones de la ciudad por parte de estos actores que, en definitiva, son quienes construyen y conforman la ciudad.

BUENOS AIRES (ARGENTINA), MARZO 2004

\section{BIBLIOGRAFÍA}

CiCCOLELla, P. e I. MignAQUi (2000): «Distribución global y territorio. Modernización y concentración comercial en Argentina en los noventa». Economía, Sociedad y Territorio, Vol. II, Nº ${ }^{\circ}$. México: El Colegio Mexiquense.

CEPAL (2000): Juventud, población y desarrollo. Problemas, posibilidades y desafíos. Santiago: CEPAL.

FERNÁNDEZ, GABRIEL (2003): «El acceso a la vivienda de los jóvenes en la zona sur de la región metropolitana de Buenos Aires: la cuestión habitacional en grupos específicos». Geocrítica, Scripta Nova, Revista Electrónica de Geografía y Ciencias Sociales, Vol. VII, N¹46. Barcelona: Universidad de Barcelona.

INDEC (1999): Anuario estadístico 1999. Encuestas juveniles en temas sociales. Buenos Aires: INDEC.

MATURANA, H. (1999): «La participación como necesidad». Cuadernos de Participación del FOSIS. Santiago: FOSIS.

PíREZ, P. (1998): «Actores sociales y gestión de la ciudad». Ciudades, Red Nacional de Investigación Urbana, Año 7, №28.

- (1994): Gobierno y gestión de la ciudad, Buenos Aires metropolitana. Buenos Aires: Centro Editor de América Latina.

RoDRíGUEZ, M. C. (2002): «Producción social del hábitat, cooperativismo autogestionario y derecho a la ciudad. Reflexiones desde la ciudad de Buenos Aires». Mundo Urbano No17.

YuJNOVSKY, O. (1984): Claves políticas del problema habitacional argentino, 1955-1981. Buenos Aires: Grupo Editor Latinoamericano. 